\title{
Streamwise streaks induced by bedload diffusion
}

\author{
Anaïs Abramian ${ }^{1} \dagger$, Olivier Devauchelle ${ }^{1}$ and Eric Lajeunesse ${ }^{1}$ \\ ${ }^{1}$ Institut de Physique du Globe de Paris, France \\ (Received xx; revised xx; accepted xx)
}

\begin{abstract}
A fluid flowing over a granular bed can move its superficial grains, and eventually deform it by erosion and deposition. This coupling generates a beautiful variety of patterns such as ripples, bars and streamwise streaks. Here, we investigate the latter, sometimes called "sand ridges" or "sand ribbons". We perturb a sediment bed with sinusoidal streaks, the crests of which are aligned with the flow. We find that, when their wavelength is much larger than the flow depth, bedload diffusion brings mobile grains from troughs, where they are more numerous, to crests. Surprisingly, gravity can only counter this destabilising mechanism when sediment transport is intense enough. Relaxing the long-wavelength approximation, we find that the cross-stream diffusion of momentum mitigates the influence of the bed perturbation on the flow, and even reverses it for short wavelengths. Viscosity thus opposes the diffusion of entrained grains to select the most unstable wavelength. This instability might turn single-thread alluvial rivers into braided channels.
\end{abstract}

Key words: Bedload transport, bedforms, pattern formation, river morphology, granular diffusion.

\section{Introduction}

When water flows over a granular bed with enough strength, it dislodges some of the superficial grains and entrains them downstream (Shields 1936; Einstein 1937; Bagnold 1973). As long as the flow-induced force is comparable to their weight, the entrained grains remain close to the bed surface, where they travel with the flow, until they eventually settle down. In steady state, the balance between entrainment and settling sets the number of travelling grains, which thus depends primarily on the flow-induced shear stress (Charru et al. 2004a; Lajeunesse et al. 2010). Accordingly, the sediment flux resulting from their collective motion, called bedload transport, is usually expressed as a function of shear stress (Meyer-Peter \& Müller 1948).

Bedload transport is often heterogeneous - it scours away the bed somewhere, and deposits the entrained material elsewhere (Exner 1925). The flow then adjusts to the deformed bed, and alters the distribution of erosion and deposition. This fluid-structure interaction generates bedforms through various instabilities (Seminara 2010; Charru et al. 2013).

Current ripples are iconic underwater bedforms found in streams, on beaches and sometimes in the sedimentary record (Allen 1982; Coleman \& Melville 1994). They result from the inertia of the flow, which concentrates shear stress just upstream of their crest

$\dagger$ Email address for correspondence: anaisabramian@gmail.com 


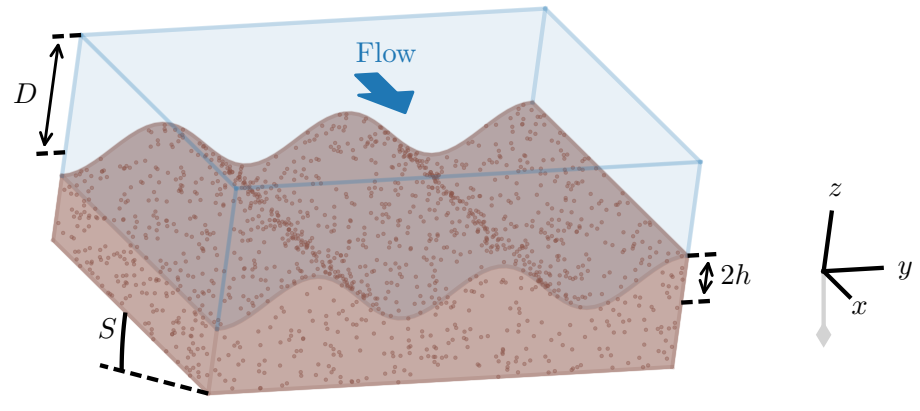

FiguRE 1. Sediment bed perturbed by longitudinal streaks. A layer of fluid (blue) flows over a granular bed (brown). The reference frame is inclined with respect to gravity (downstream slope S). The vertical grey line with a diamond marker symbolises a plumb line.

(Kennedy 1963; Charru 2006; Charru et al. 2013). Nascent ripples make the most of this mechanism by orienting their crest across the flow. At least initially, they do not involve any cross-stream sediment flux. By contrast, the oblique crest of alternate bars diverts the water flow to induce the cross-stream bedload flux that makes them unstable (Parker 1976; Colombini et al. 1987; Devauchelle et al. 2010; Andreotti et al. 2012).

Although less common, streamwise streaks materialise cross-stream bedload more neatly - their crest remains aligned with the stream as they grow (Karcz 1967; Colombini \& Parker 1995; McLelland et al. 1999). To initiate a cross-stream flux of sediment, these bedforms use a subtle peculiarity of turbulence. When streamwise ridges perturb their boundary, turbulent flows generates transverse, counter-rotating vortices (Colombini 1993; Vanderwel \& Ganapathisubramani 2015). Over a granular bed, these slow secondary currents transport sediment across the primary flow to accumulate it in upwelling areas, thus reinforcing the ridges that brought them about. A similar phenomenon occurs when grains of different sizes make up the bed, the heterogeneous roughness of which then plays the role of ridges (McLelland et al. 1999; Willingham et al. 2014).

In the above examples, the sediment grains travel along the force that entrains them. This is certainly true on average, but bedload particles slide and roll over a rough bed, which makes their trajectory seesaw across the stream (Nikora et al. 2002; Furbish et al. 2012). The experiments of Seizilles et al. (2014) show that these fluctuations cause the grains to disperse laterally, like random walkers. Collectively, they diffuse across the bedload layer towards areas of lesser transport, thus moving across the stream in the absence of transverse flow. We speculate that this Fickian diffusion could create streamwise streaks without secondary currents, provided bedload is less intense on the bedforms' crests.

To test this scenario, we investigate the stability of a flat sediment bed sheared by a laminar flow. We begin with the shallow-water approximation (section 2). Extending our analysis to two dimensions, we then find that viscosity, which diffuses momentum across the stream, selects the size of the most unstable mode (section 3). We then consider a stream covered with a rigid lid, which might facilitate measurements in a laboratory experiment (section 4). Finally, we look for this instability in previous experiments (section 5). 


\section{Bedload instability}

\subsection{Base state}

We consider an infinitely wide, flat granular bed sheared by a free-surface, laminar flow (figure 1). A small slope $S$ drives the fluid along $x$, the streamwise direction, but we will neglect its effect on the weight of a grain later on. We further assume that the size of a grain, $d_{s}$, is much smaller than the flow depth $D$. In steady state, the shear stress the fluid exerts on the bed, $\tau$, is the projection of its weight on the streamwise direction:

$$
\tau=\rho g D S
$$

where $\rho$ is the density of the fluid, and $g$ the acceleration of gravity.

To entrain a grain, the fluid needs to pull it with enough strength to overcome its weight. Mathematically, this happens when the ratio of these two forces, $\theta$, exceeds a threshold value, $\theta_{t}$. Shields (1936) defined this ratio as

$$
\theta=\frac{\tau}{\left(\rho_{s}-\rho\right) g d_{s}}
$$

where $\rho_{s}$ is the density of a grain.

Based on laboratory observations, Charru et al. (2004a) suggested that the number of grains the fluid dislodges from the bed, per unit surface and time, is proportional to the distance to threshold, $\theta-\theta_{t}$. The bedload layer is fed by this constant input. Conversely, it loses a fraction of its population through settling. When moving grains are too sparse to interact, the settling rate is proportional to the number of moving grains per unit area, $n$ (Aussillous et al. 2016). At equilibrium, the density of moving grains thus reads

$$
n=\frac{\alpha_{n}}{d_{s}^{2}}\left(\theta-\theta_{t}\right)
$$

where $\alpha_{n}$ is, like $\theta_{t}$, a dimensionless, empirical parameter (Lajeunesse et al. 2010). For illustration, $\theta_{t} \sim 0.1$ and $\alpha_{n} \sim 0.01$ are typical values for these parameters in a laminar flow (Seizilles et al. 2014). Equation (2.3) is valid only above threshold, that is, when $\theta \geqslant \theta_{t}$; below threshold, the bedload layer is empty $(n=0)$.

After equation (2.3), a flow near threshold can only entrain a sparse bedload layer. Then, the velocity of the travelling grains is that of the fluid near the bed, which is proportional to shear stress in a laminar flow (Seizilles et al. 2014). As a consequence, the average velocity in the bedload layer is proportional to Stokes' settling velocity, which reads

$$
V_{s}=\frac{\left(\rho_{s}-\rho\right) g d_{s}^{2}}{18 \eta}
$$

where $\eta$ is the viscosity of the fluid. The sediment flux, $q_{s}$, results from the collective motion of the bedload grains:

$$
q_{s}=\alpha_{v} n V_{s}
$$

where $\alpha_{v}$ is a dimensionless coefficient. In a laminar flow, Seizilles et al. (2014) found $\alpha_{v} \sim 0.4$.

Most authors relate bedload directly to the Shields parameter with a sediment transport law (Meyer-Peter \& Müller 1948). Combining equations (2.3) and (2.5), we find that bedload transport is proportional to the distance to threshold. The specific expression of this law, however, is still debated, and is likely to depend on the particle Reynolds number (Ouriemi et al. 2009). Here, we choose a simple law that compares reasonably with near-threshold, laminar experiments (Charru et al. 2004a; Seizilles et al. 2014).

Equations (2.1) to (2.5) represent a uniform base state, both in the downstream 
a
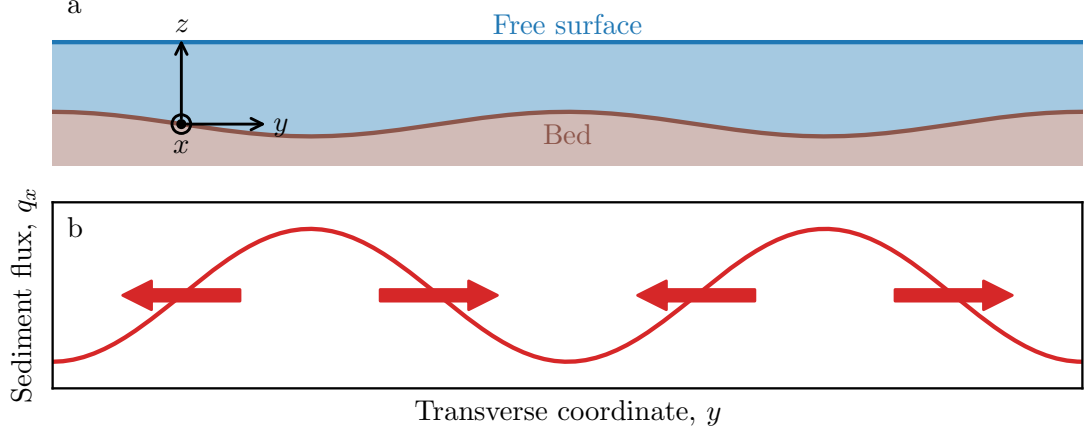

Figure 2. Mechanism of the bedload instability. a: Bed elevation (brown) and free surface flow (blue). b: Distribution of the corresponding downstream sediment flux. Red arrows indicate bedload diffusion.

direction $x$ and in the cross-stream direction $y$. In the following, we add a perturbation to it to introduce bedload diffusion.

\subsection{Bedload diffusion}

Heterogeneity drives diffusion. To introduce some of it in our system, we now carve streamwise streaks into the granular bed, in the form of a sinusoidal perturbation of amplitude $h$ and wavelength $\lambda$ (figure 1). The fluid flow and the sediment bed remain invariant along $x$, and our system is now two-dimensional.

To illustrate the mechanism of bedload instability, we consider, in this section, that the amplitude of the perturbation is much smaller, and its wavelength much longer, than the flow depth. With these assumptions, we expect the shallow-water approximation to yield a reasonable estimate of the shear stress $\tau$, and therefore of the Shields parameter $\theta$. Both are then proportional to the local flow depth, $D-h$, and therefore of lesser intensity at the crest of the perturbation. Mathematically,

$$
\theta=\frac{\rho D S}{\left(\rho_{s}-\rho\right) d_{s}}\left(1-\frac{h}{D}\right) .
$$

According to equation (2.3), the bedload layer is thus denser in the troughs than on the crests. Its density reads

$$
n=n_{0}-\frac{\alpha_{n} \theta_{0}}{d_{s}^{2}} \frac{h}{D}
$$

where $n_{0}$ and $\theta_{0}$ are the density of moving grains and the Shields parameter in the base state, respectively. Like equation (2.3), from which it is derived, the above equation only holds above threshold, that is, when

$$
\frac{h}{D} \leqslant \frac{\theta_{0}-\theta_{t}}{\theta_{0}}
$$

This condition sets the maximum amplitude the perturbation $h$ can reach before the following analysis breaks down.

Following Seizilles et al. (2014), we now treat the bedload grains as independent random walkers. As they travel downstream at the average velocity $\alpha_{v} V_{s}$, their cross-stream velocity fluctuates around zero. We represent this process by a series of random sideways steps, the amplitude of which is a fraction of the grain size. Statistically, the accumulation 
of these steps generates a diffusive flux, $q_{d}$, towards the less populated areas of the bedload layer. As long as their density remains low, the moving grains do not interact with each other, and bedload diffusion obeys Fick's law:

$$
q_{d}=-\alpha_{v} \ell_{d} V_{s} \frac{\partial n}{\partial y}
$$

where we expect the diffusion length, $\ell_{d}$, to be a fraction of the grain size. Seizilles et al. (2014) found $\ell_{d} \sim 0.03 d_{s}$ in their laminar experiment, and we are not aware of any other measurement of this empirical parameter.

The stage is now set for the bedload instability: diffusion brings grains from the troughs, where bedload is more intense, to the less populated crests, thus furrowing further into the bed (figure 2). This mechanism, however, is countered by gravity, to which we now turn our attention.

\subsection{Stability}

As a grain travels over a slanting bed, gravity deflects its trajectory downwards. For an arbitrary slope, the mathematical formulation of the resulting cross-stream flux is intricate, and its experimental evaluation challenging (Kovacs \& Parker 1994). Fortunately, for a linear stability analysis, we may content ourselves with a first-order formulation valid for shallow slopes, and near the threshold of motion. Accordingly, we assume that the cross-stream, slope-induced flux, $q_{g}$, is proportional to the density of moving grains, and to the cross-stream slope of the bed:

$$
q_{g}=-\gamma \alpha_{v} V_{s} n \frac{\partial h}{\partial y}
$$

where $\gamma$ is another empirical parameter - the last we will need. Based on the observations of Yamasaka et al. (1987) in a wind tunnel, Chen et al. (2009) proposed that $\gamma$ lies in the range $0.1-1$. Most probably, this value depends on the Reynolds number at the grain's scale, and on the relative density of the grain. To our knowledge, it has never been measured in a laminar flow.

The gravity flux, $q_{g}$, brings bedload grains from crest to trough, and thus hinders the instability driven by the diffusion flux, $q_{d}$. Mass balance mediates this competition through the Exner equation:

$$
\frac{\partial h}{\partial t}+\frac{d_{s}^{3}}{\phi} \frac{\partial}{\partial y}\left(q_{d}+q_{g}\right)=0
$$

where $\phi$ and $t$ are the packing fraction of the sediment bed and time, respectively. For randomly packed spheres, the packing fraction is approximately $\phi \sim 0.6$ (Bernal \& Mason 1960). The bedload density equation (2.7), the cross-stream flux equations (2.9) and (2.10), and the the Exner equation form a closed system, which the slope-induced flux makes nonlinear.

We consider an infinitesimal perturbation of the bed, and expand our equations up to first order in its amplitude. When we combine its four equations, the resulting linear system takes the familiar form of the heat equation:

$$
\frac{\partial h}{\partial t}-\frac{q_{s 0} d_{s}^{3}}{\phi}\left(\gamma-\frac{\ell_{d}}{D} \frac{\theta_{0}}{\theta_{0}-\theta_{t}}\right) \frac{\partial^{2} h}{\partial y^{2}}=0
$$

where $q_{s 0}$ is the bedload intensity of the base state. The diffusivity of this equation depends on the base state, and indeed can change sign. Its expression straightforwardly conveys the character of the two cross-stream fluxes. The slope-induced flux, represented 


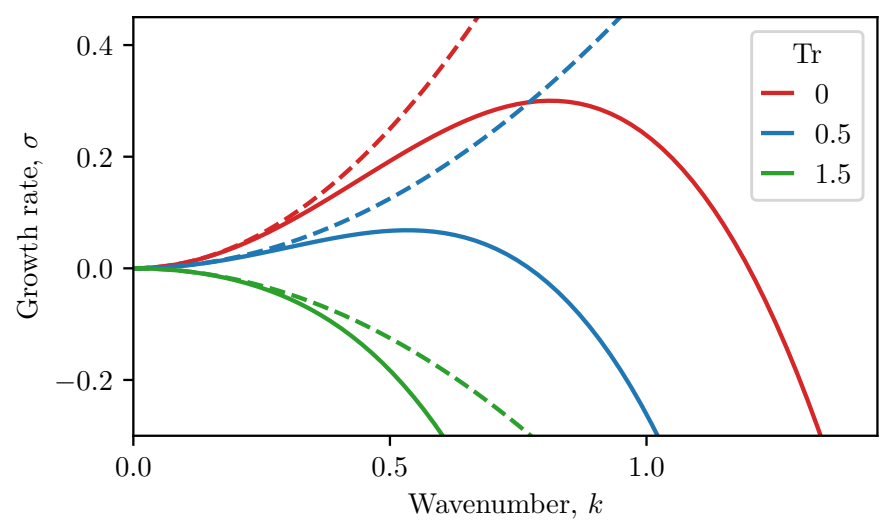

Figure 3. Dispersion relation of the bedload instability, in a free-surface flow. Dashed lines: shallow-water approximation (equation (2.17)). Solid lines: two-dimensional Stokes flow (equation (3.9)).

by $\gamma$, tends to increase the diffusivity, and thus stabilises the system. Conversely, the diffusive flux, represented by $\ell_{d}$, destabilises the bed - as anticipated, bedload diffusion drives the instability.

We now introduce the transport number, Tr, as the ratio of the two terms of the diffusivity in equation (2.12):

$$
\operatorname{Tr}=\frac{\gamma D}{\ell_{d}} \frac{\theta_{0}-\theta_{t}}{\theta_{0}}
$$

Combining this definition with the bedload transport law (equation (2.5)) recasts the transport number into a dimensionless sediment flux:

$$
\operatorname{Tr}=\frac{\gamma D d_{s}^{2}}{\alpha_{v} \alpha_{n} \ell_{d} V_{s}} \frac{q_{s 0}}{\theta_{0}} .
$$

Literally, Tr depends not only on the sediment flux of the base state, but also on the Shields parameter, $\theta_{0}$. Near the threshold, however, $\theta_{t}$ becomes a good approximation for $\theta_{0}$; the transport number then bears its name rightly.

When bedload is intense in the base state, the transport number is large, and so is diffusivity in equation (2.12). Sediment transport therefore stabilises the bed. Conversely, a vanishing sediment flux brings the transport number arbitrarily close to zero. Eventually, the effective diffusivity in equation (2.12) becomes negative, and bedload diffusion destabilises the bed.

This surprising result can be understood as follows. After the shallow-water approximation, the density of moving grains is simply proportional to the local depth (equation (2.7)). As a consequence, its cross-stream gradient does not depend on the intensity of bedload in the base state. Conversely, the slope-induced flux is proportional to $n_{0}$, and therefore to the downstream sediment flux in the base state. Intense bedload thus favours the stabilising mechanism, without affecting bedload diffusion.

To formalise this discussion, we now perform the linear stability analysis of equation (2.12), an elementary undertaking that will serve as a guide for the next section. We first introduce the following characteristic time:

$$
T=\frac{\phi D^{3}}{\theta_{0} \alpha_{n} \alpha_{v} V_{s} \ell_{d} d_{s}}
$$


which, when lengths are rescaled with $D$, makes equation (2.12) dimensionless. Next, we express the bed perturbation as a sinusoidal wave of dimensionless amplitude $\hat{h}$ :

$$
\frac{h}{D}=\operatorname{Re}\left(\hat{h} \exp \left(i k \frac{y}{D}+\sigma \frac{t}{T}\right)\right),
$$

where $k$ and $\sigma$ are the dimensionless wavenumber and growth rate of the perturbation, respectively. Substituting this expression in equation (2.12) yields the dispersion relation of the instability:

$$
\sigma=k^{2}(1-\operatorname{Tr})
$$

When the base state transports enough sediment to bring the transport number above one, the growth rate is negative for any wavenumber, indicating that all perturbations of the bed decay (figure 3). This oversimplified model transitions to instability drastically: all wavelengths suddenly become unstable as the transport number is reduced below one. Short-wavelength perturbations grow infinitely fast, and equation (2.12) is then ill-posed.

In reality, of course, streamwise streaks will never grow infinitely fast. This mathematical exaggeration is only due to some rash assumption. In the next section, we attempt to fix our model by dropping the shallow-water approximation.

\section{Regularisation by cross-stream diffusion of momentum}

\subsection{Stokes flow}

Bedload diffusion reinforces streamwise streaks provided (i) bedload transport is weak, and (ii) the flow exerts a lower shear stress on the crests than in the troughs. When the wavelength of the perturbation is much longer than the flow depth, the shallowwater approximation ensures that the latter condition is fulfilled. For small wavelengths, however, viscosity diffuses momentum in the cross-stream direction (y), thus dampening the influence of the bed perturbation on the flow. This mechanism regularises the instability. By definition, the shallow-water approximation neglects this mechanism, and unduly maintains the instability for short wavelengths. In this section, we replace the shallow-water approximation with a two-dimensional Stokes equation.

We still consider a streamwise-invariant, laminar flow. However, the downstream velocity $u$ is now a function of $y$ and $z$ (figure 2). This Stokes flow then obeys Poisson's equation:

$$
\eta \nabla^{2} u=-\rho g S
$$

where $\eta$ is the viscosity of the fluid, and the Laplacian operator $\nabla^{2}$ applies to crossstream coordinates only. We further assume that the flow does not slip along the bed surface, and that the fluid surface is free of constraint. Mathematically,

$$
u=0 \quad \text { at } \quad z=h
$$

and

$$
\frac{\partial u}{\partial z}=0 \quad \text { at } \quad z=D
$$

Solving Poisson's equation with the above boundary conditions yields the shear stress the flow exerts on the bed surface:

$$
\tau=\eta \mathbf{e}_{n} \cdot \nabla u \quad \text { at } \quad z=h
$$

where $\mathbf{e}_{n}$ is the unit vector normal to the bed surface.

Like in section 2, the flow entrains superficial grains as bedload, thus altering the bed 
surface through erosion and deposition. Here, however, this coupling takes the form of a free-boundary problem in two dimensions, since the Exner equation transforms the domain over which Poisson's equation is to be solved. Fortunately, linearising makes its stability analytically tractable.

Let us first consider the velocity profile of the base state, $u_{0}$. Like the bed itself, it is invariant along the streamwise and cross-stream direction, and Poisson's equation reduces to the direction normal to the bed, $z$. Its solution is half the classical parabola of the Poiseuille flow:

$$
u_{0}=9 \frac{\theta_{0} V_{s} D}{d_{s}}\left(2 \frac{z}{D}-\left(\frac{z}{D}\right)^{2}\right)
$$

which provides us with a velocity scale for the flow, namely $\theta_{0} V_{s} D / d_{s}$.

The velocity profile of the base state equates the source term of Poisson's equation. Therefore, the velocity perturbation is a solution of Laplace's equation or, equivalently, it is the real part of some analytical function of the complex coordinate $y+i z$. This velocity profile vanishes at the surface of the bed (equation (3.2)), the elevation of which is perturbed by sinusoidal streaks (equation (2.16) at $t=0$ ). In addition, its normal derivative also vanishes at the surface of the flow or, equivalently, it is symmetrical with respect to this surface (equation (3.3)). At first order, we find:

$$
u=u_{0}-18 \frac{\theta_{0} V_{s} D}{d_{s}} \operatorname{Re}\left(\frac{\hat{h}}{\cosh (k)} \cosh \left(i k \frac{y+i z}{D}+k\right)\right) .
$$

Figure 4 shows the iso-velocity lines of the first-order flow, that is, the contours of the above expression. When the wavelength of the perturbation is much larger than the flow depth $(\lambda \gg D$ or, equivalently, $k \ll 1)$, the Stokes flow accords qualitatively with the shallow-water approximation: its contours constrict above troughs, indicating intense shear stress on the deepest parts of the bed. This distribution of shear stress favours the instability.

The iso-velocity lines tell a different story for short wavelengths $(k \gg 1)$. As the space between streaks gets narrower, the bulk of the flow cannot squeeze in between crests any more. The latter then find themselves fully exposed to the flow, from which they shelter the troughs. As a result, shear stress is more intense on the crests than in the troughs, and bedload diffusion will combine with gravity to wear the streaks down. We thus anticipate that the screening induced by the diffusion of momentum across the flow, which is the signature of Laplace's equation, will provide us with the regularisation we lacked in section 2 .

\subsection{Dispersion relation}

To turn the above reasoning into a quantitative expression, we first need to calculate the shear stress on the sediment bed. Deriving equation (3.6) with respect to $z$, and evaluating the result on the bed yields the bed shear stress, and therefore the Shields parameter, at first order:

$$
\theta=\theta_{0}\left(1-(1-k \tanh k) \operatorname{Re}\left(\hat{h} \exp \left(\frac{i k y}{D}\right)\right)\right)
$$

According to this expression, the perturbation of the Shields parameter is either in phase or in antiphase with the bed perturbation, depending on wavelength (figure 4). For long wavelengths $(k \ll 1)$, the Shields parameter approaches its shallow-water expression (equation (2.6)); it then reaches its maximum above a trough, and the bed is unstable. 

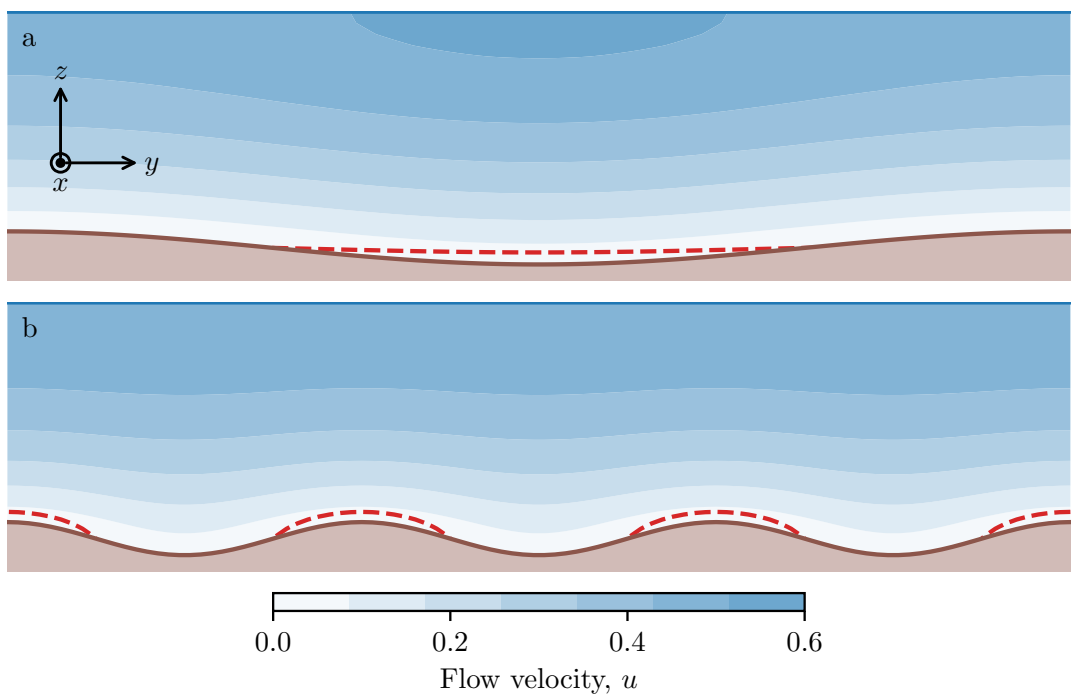

FiguRE 4. Stokes flow above streamwise streaks. Bed elevation (brown) and iso-velocity contours (blue scale). Below the red dashed line, the shear stress is higher than in the base state. a: long wavelength $(k D=1.4)$. b: short wavelength $(k D=4.2)$.

Conversely, for short wavelengths $(k \gg 1)$, equation $(3.7)$ reduces to

$$
\theta \approx \theta_{0}\left(1+k \operatorname{Re}\left(\hat{h} \exp \left(\frac{i k y}{D}\right)\right)\right),
$$

which reverses the phase of the Shields parameter with respect to the bed perturbation. The shear stress then concentrates on the crests, thus stabilising the bed.

Equipped with a new expression for the Shields parameter, we now return to the stability analysis of section 2. We substitute equation (2.6) for equation (3.7), and reuse the expression of the bedload fluxes, which the new flow model leaves unaffected (equations (2.3), (2.9) and (2.10)). Injecting these equations into the mass-balance equation (2.11) yields a new dispersion relation:

$$
\sigma=k^{2}(1-\operatorname{Tr})-k^{3} \tanh k .
$$

This expression equals its shallow-water counterpart (equation 2.17), but for an additional regularisation term. The latter, always negative, dominates the dispersion relation at short wavelength (large $k$ ). Thanks to the two-dimensional Stokes flow, equation (3.9) now behaves like a proper dispersion relation - the growth rate never diverges (figure 3 ).

The transport number still controls the global stability of the bed. Intense sediment transport $(\operatorname{Tr}>1)$ precludes the bedload instability. When the transport number becomes less than its critical value of one, however, a range of unstable wavelengths appears. Within this range, a well-defined maximum indicates the most unstable mode, which is likely to emerge in experiments.

Figure $5 \mathrm{a}$ shows the trajectory of the most unstable mode on a stability map. As the transport number crosses its critical value, the bedload instability appears with a vanishing wavenumber, which quickly increases. The wavenumber of the most unstable mode then increases towards its maximum value $k_{\max }$, which it reaches when the base state transports no sediment $(\operatorname{Tr}=0)$. The largest of all most unstable wavenumber, 

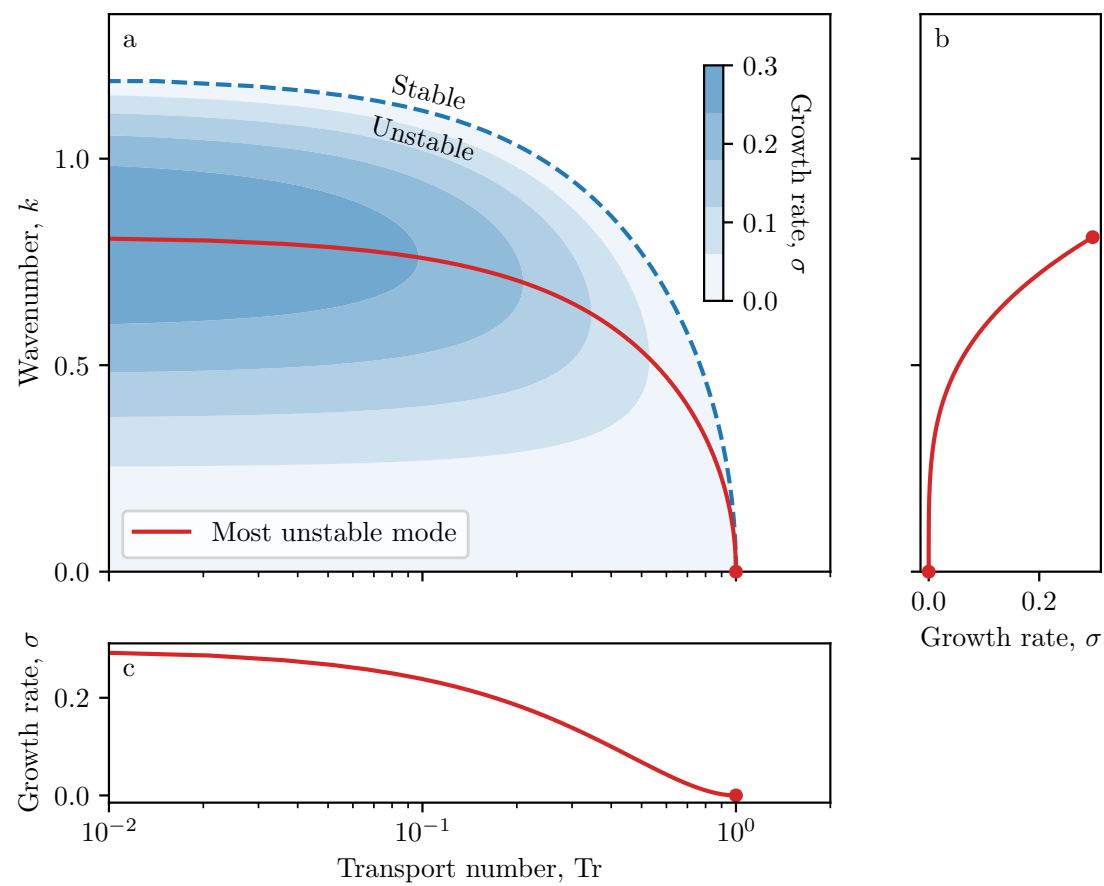

Growth rate, $\sigma$

FiguRE 5. Stability map of the bedload instability, for a free surface flow. a: Dispersion relation. Darker areas correspond to faster growth rate. Red line: most unstable wave number for a given value of the transport number. b: Growth rate of the most unstable wavenumber. c: Maximum growth rate as a function of the transport number.

$k_{\text {max }}$, is the positive solution of the following equation:

$$
3 k \tanh (k)+k^{2}\left(1-\tanh ^{2} k\right)=2 .
$$

Numerically, $k_{\max } \approx 0.813$. Returning to dimensional quantities, we find that $k_{\max }$ translates into a remarkably universal ratio. When the intensity of bedload vanishes, the most unstable wavelength, $\lambda_{\max }$, is simply proportional to the flow depth, regardless of any other parameter:

$$
\frac{\lambda_{\max }}{D}=\frac{2 \pi}{k_{\max }} \approx 7.73
$$

This result suggests a recipe for an experiment - a hypothetical one for now. To identify the bedload instability unambiguously, one need only reduce the bedload flux until the wavelength of the instability reaches its minimum, which should be the above value. Unfortunately, laboratory experiments seldom abide by idealised theories; we will return to this question in the discussion (Section 5).

In addition to a wavelength, the most unstable mode also has a growth rate, $\sigma_{\max } \approx$ 0.300 , which we calculate by injecting $k_{\max }$ into the dispersion relation, and sending the transport number to zero. Like the wavenumber of the most unstable mode, the growth rate is at its highest when the base state transports no sediment ( $\operatorname{Tr}=0$, figure $5 \mathrm{c}$ ). This statement, counter-intuitive with dimensionless quantities, becomes preposterous in physical units: after equation (2.15), the dimensional growth rate also reaches its 


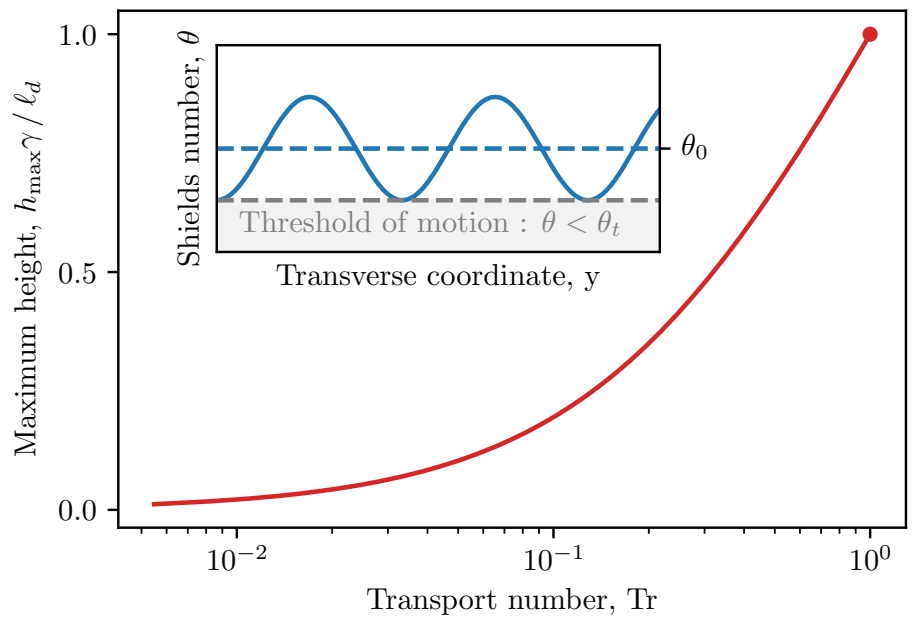

FiguRE 6. Saturation of the bedload instability. Red line: amplitude of the bed perturbation at the transition to the non-linear regime, $h_{\max }$, as a function of the transport number. Inset : transition criterium, the amplitude of the perturbation of the Shields parameter reaches $\theta_{0}-\theta_{t}$.

maximum when bedload transport vanishes. Since it is bedload that feeds the instability, we would expect its absence to turn off the growth of the streaks. This apparent paradox is due to the threshold of the sediment transport law (equation (2.3)), which brings the linear regime to its end.

\subsection{End of the linear regime}

As the flow-induced shear stress approaches the threshold, bedload vanishes from the base state, and so does the efficiency of the gravity-induced flux. This, however, does not affect the sensitivity of bedload, which responds as strongly as ever to any perturbation of the flow. Therefore, while the stabilising mechanism vanishes, the unstable coupling between the flow and the bed persists - the bedload instability then thrives.

Such full-blown growth, however, will quickly meet its end. The linear expansion of the transport law holds as long as the perturbed bed remains above the threshold. This gets more demanding as the base state itself approaches the threshold (figure 6). We define $h_{\max }$ as the amplitude of the perturbation when the Shields parameter hits the threshold. Mathematically, after equation (3.7), $h_{\max }$ reads:

$$
h_{\max }=\frac{\ell_{d}}{\gamma} \frac{\operatorname{Tr}}{1-k \tanh k} .
$$

For any value of the transport number Tr, we calculate numerically the wavenumber $k$ of the most unstable mode, and inject the result in the above expression (figure 6). We find that, as the intensity of bedload vanishes $(\mathrm{Tr} \rightarrow 0$ ), so does the maximum amplitude of the most unstable mode. Our linear stability analysis then holds for imperceptible streaks only.

Once the perturbation has outgrown $h_{\max }$, the instability enters a nonlinear regime, to which the present analysis grants no access. We speculate, however, that the perturbation could keep growing beyond this point, since its driving mechanism - active troughs and frozen crests - should survive the end of linearity. 


\section{Rigid lid}

Although most bedload experiments involve open channels, the free surface of the flow makes the tracking of sediment grains difficult. To avoid this problem, some experimenters covered the flow with a transparent lid (Charru et al. 2004a; Lobkovsky et al. 2008; Aussillous et al. 2016). In this section, we investigate the influence of this new boundary condition on the bedload instability.

\subsection{Fixed lid}

Like in the experiments of Lobkovsky et al. (2008), a fixed, flat and rigid lid now confines the fluid above the sediment bed. The flow is driven along this infinitely wide pipe either by a downstream pressure gradient or, like in the previous sections, by the tilt of the bed. Equating the pressure gradient with $\rho g S$, we may treat these configurations alike.

Following the structure of section 2, let us first use the shallow-water approximation, keeping its limitations in mind. Above a long-wavelength perturbation, the flow profile is essentially the Poiseuille parabola - the entire one, this time. It is symmetric with respect to the centre of the fluid gap, and therefore distributes shear stress evenly between the lid and the bed. We thus replace equation (2.6) by

$$
\theta=\frac{\rho D S}{2\left(\rho_{s}-\rho\right) d_{s}}\left(1-\frac{h}{D}\right)
$$

Apart from the factor of 2 in the above expression, the lid does not affect our system, at least according to the shallow-water approximation. This factor of 2 does not even propagate beyond equation (2.3) where, by virtue of the normalisation by $n_{0}$ and $\theta_{0}$, it retreats into the definition of the base state. We are thus left with equation (2.17) again, that is, the same dispersion relation as in section 2 .

Based on the above reasoning, we expect the lid to affect the bedload instability only marginally, if at all. To check this formally, we now repeat the two-dimensional stability analysis of section 3, with a new boundary condition at the flow surface. Specifically, we replace the free-boundary condition with a no-slip one. At first order, we find the following velocity field:

$$
u=18 \frac{\theta_{0} V_{s} D}{d_{s}}\left(\frac{z}{D}\left(1-\frac{z}{D}\right)-\operatorname{Re}\left(\frac{\hat{h}}{\sinh k} \sinh \left(i k \frac{y+i z}{D}+k\right)\right)\right)
$$

which replaces equation (3.6). The dispersion relation associated with this expression reads

$$
\sigma=k^{2}(2-\operatorname{Tr})-\frac{k^{3}}{\tanh k},
$$

which, qualitatively, behaves like its open-channel counterpart, equation (3.9). Again, the bed is stable when the transport number is larger than one, and the dispersion relation shows a positive maximum otherwise. The most unstable mode grows faster as bedload vanishes $(\operatorname{Tr} \rightarrow 0)$, until the growth rate reaches is maximum $\left(\sigma_{\max } \approx 0.831\right)$, for the mode of wavelength $\lambda_{\max } / D \approx 4.785$.

This result encourages us to look for the bedload instability in a closed channel, where their observation should be easier than in an open one - although, to our knowledge they have never been reported in any laboratory experiment. 


\subsection{Driving lid}

In their experiment, Charru et al. (2004b) drove the flow along a circular channel with a rotating lid. The ring circumference was much larger that the channel's width; we now approximate this configuration with an infinitely long channel, covered with a rigid lid travelling downstream at velocity $U$. The shallow-water approximation then yields

$$
\theta=\frac{\eta U}{D\left(\rho_{s}-\rho\right) g d_{s}}\left(1+\frac{h}{D}\right) .
$$

Unlike equations (2.6) and (4.1), equation (4.4) indicates that the shear stress is stronger where the bed is closer to the moving lid, that is, at the crest of the perturbation. This is a dramatic change: bedload diffusion and gravity now conspire to wear away even a long-wavelength perturbation.

The two-dimensional velocity field now reads

$$
u=18 \frac{\theta_{0} V_{s} D}{d_{s}}\left(\frac{z}{D}-\operatorname{Re}\left(\frac{\hat{h}}{\sinh (k)} \sinh \left(i k \frac{y+i z}{D}+k\right)\right)\right),
$$

and the associated Shields parameter becomes

$$
\theta=\theta_{0}\left(1+\left(\frac{k}{\tanh (k)}\right) \operatorname{Re}\left(\hat{h} \exp \left(\frac{i k y}{D}\right)\right)\right)
$$

thus confirming that, regardless of the wavenumber $k$, the shear stress is always stronger at the crest. As a consequence, driving the flow with a moving lid prevents the growth of the bedload instability. Experiments like that of Charru et al. (2004b) are therefore specially well suited to produce wide and shallow flows with stable bedload transport.

\section{Laboratory channels}

Bedload experiments in laminar channels or pipes are not uncommon (Charru et al. 2004b; Ouriemi et al. 2007; Lobkovsky et al. 2008; Aussillous et al. 2013; Seizilles et al. 2014), and yet streamwise bed streaks have never been reported in laminar flows, at least to our knowledge. We now use the theory of the present paper to evaluate how unstable these experiments were.

We first disqualify the experiment of Charru et al. (2004b), the driving lid of which precludes the instability (section 4.2). Next, we need to consider the sidewalls that confine the flow in an actual channel. In principle, we should treat them as no-slip boundaries, and account for them even in the base state. This, however, will produce a base state that varies in the cross-stream direction, thus complicating the analysis tremendously.

Instead, we will content ourselves with a less ambitious analysis which, we hope, will yield an order-of-magnitude interpretation of laboratory experiments. Accordingly, we replace the sidewalls with free-slip boundaries for the fluid, and impervious boundaries for the sediment. Both conditions preserve the homogeneity of the base state, to the cost of mathematical rigour.

An expected consequence of the sidewalls is to select acceptable modes (figure 7). For the perturbation to fulfill the boundary conditions at the sidewalls, its wavenumber needs to belong to a discrete set of values:

$$
k=n \pi \frac{D}{W}
$$

where $n$ is an integer, and $W$ is the width of the channel. Substituting this expression into 
Mode 1

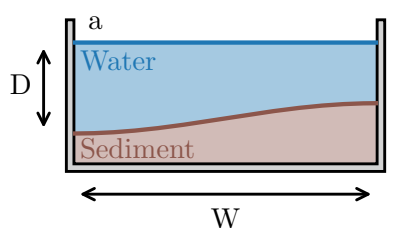

W

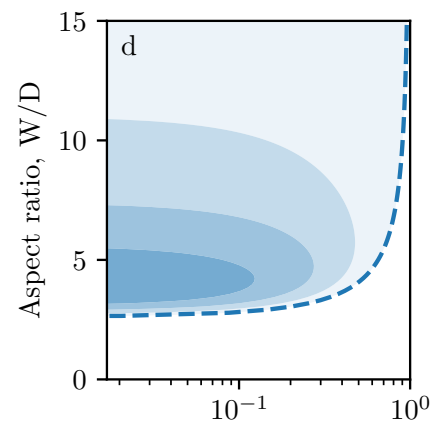

Mode 2
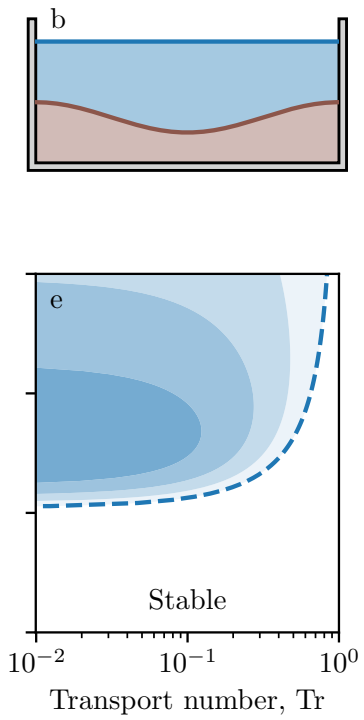

Mode 3
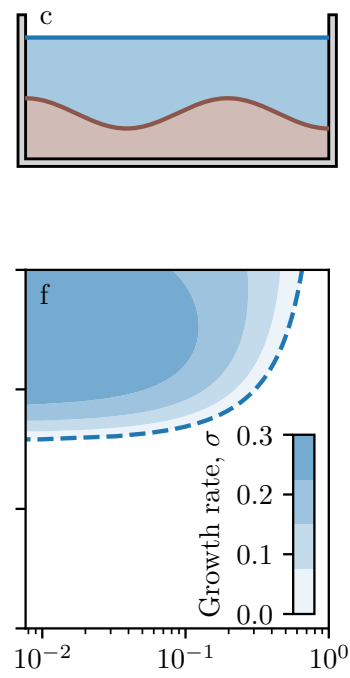

FiguRE 7. First three modes of the bedload instability in a finite-width channel. a,b,c: Bed perturbation. d,e,f: Dispersion relation for individual modes. Blue scale indicates growth rate.

the dispersion relation for an open channel, equation (3.9), we find that no instability can grow in a too narrow channel. As the transport number vanishes, the first mode $(n=1)$ is unstable only when $W / D \gtrsim 2.26$ (figure $7 \mathrm{a}$ ). The higher the mode, the wider the channel needs to be to accommodate its growth.

Considering all acceptable modes in a given experiment, we can identify the fastestgrowing one, and treat it as the dominant mode (figure 8). In the parameter space made up by the transport number and the aspect ratio, the unstable domain is tiled with individual modes. When the transport number is below one, the order of the most unstable mode increases with the aspect ratio of the channel. Overall, the unstable domain is bounded by the same curve as the first mode.

We now wish to place the laboratory experiments of Lobkovsky et al. (2008), Aussillous et al. (2013) and Seizilles et al. (2014) on the stability diagrams of figure 8. Unfortunately, we can only do this approximately since, for instance, Lobkovsky et al. (2008) and Aussillous et al. (2013) let the sediment discharge decrease slowly as the bed reaches the threshold for sediment transport. Such a transient may not last long enough for the instability to grow. Conversely, Seizilles et al. (2014) feed their open channel with a constant input of sediment, thus approaching the theory presented here. Table 1 summarises our estimates of the relevant parameters in these experiments, but many of them are uncertain. In addition, the parameter $\gamma$, which accounts for the gravityinduced flux of sediment, has never been measured in a laminar flow (section 2.3). Based on the wind-tunnel measurements of Yamasaka et al. (1987), we use the range $0.1-1$. The last parameter we need is the diffusion length $\ell_{d}$, which was measured by Seizilles et al. (2014) in a laminar flume. They found $\ell_{d} \sim 0.03 d_{s}$, which is the only estimate we can use.

Remembering that the roughness of the above estimates matches that of our simplified boundary conditions, we may now map the explored regions of the parameter space (figure 8). For the most part, they lie in the stable domain, although the experiments by 


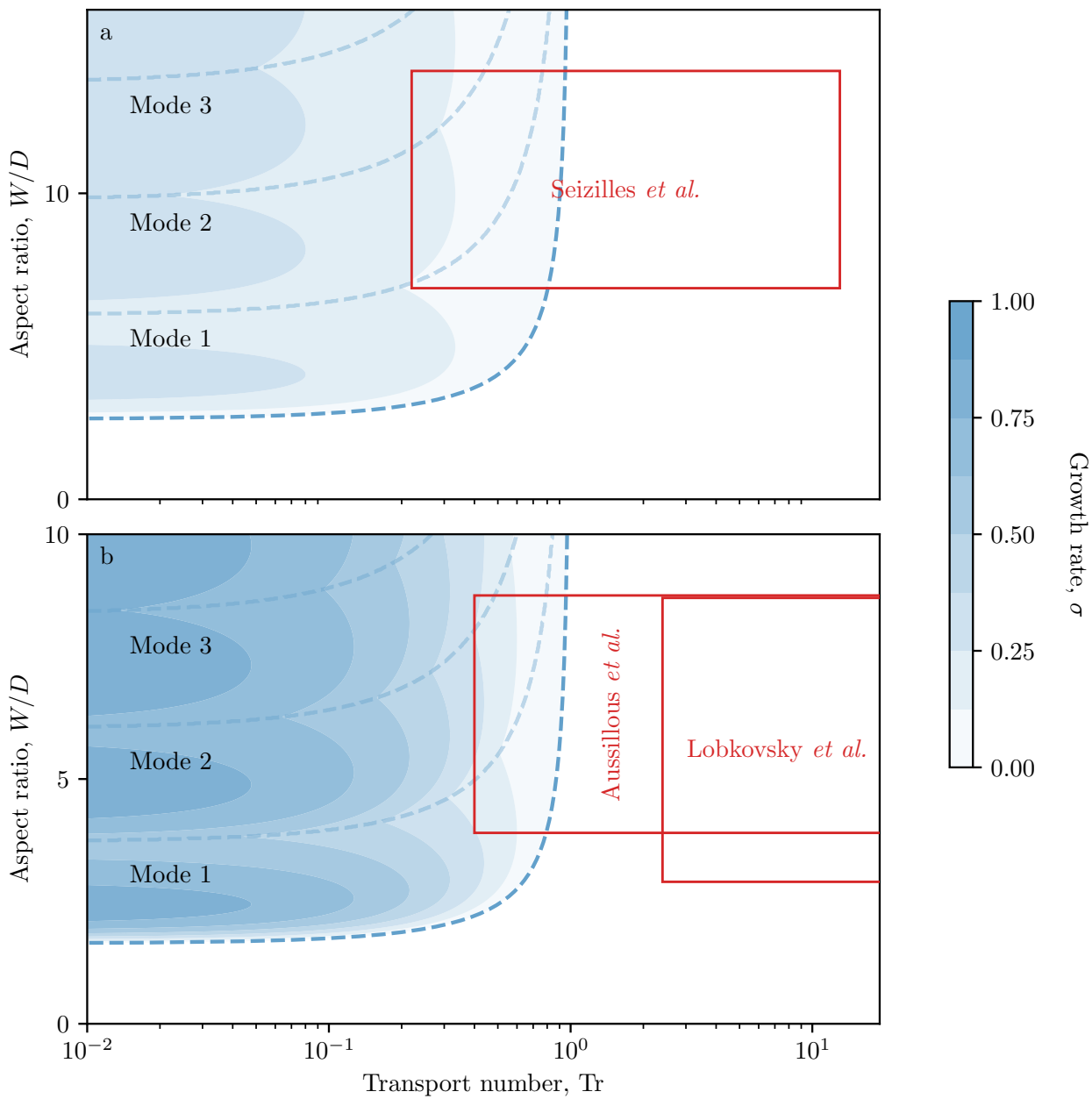

FiguRE 8. Stability map for the bedload instability in a finite-width channel. Blue scale indicates growth rate of the fastest-growing mode. Red boxes show the range of laboratory experiments. a: open channel. b: fixed lid

Aussillous et al. (2013) and Seizilles et al. (2014) marginally overlap the stable domain on the first mode. This overlap is precarious, since we neglect the influence of sidewalls on a viscous flow, in a channel whose aspect ratio is less than five.

Preliminary though it may be, figure 8 suggests that laboratory channels are usually too narrow for the bedload instability to flourish.

\section{Conclusion}

The linear stability analysis we have presented in this paper identifies a new instability associated with bedload transport, caused by the cross-stream diffusion of the travelling grains. This instability produces bed streaks aligned with the flow, in the absence of any secondary currents. Because it can only grow near the threshold of sediment transport, the unstable perturbation quickly enters a nonlinear regime which we have 
Lobkovsky et al. (2008) Aussillous et al. (2013) Seizilles et al. (2014)

\begin{tabular}{lccc}
\hline Channel & $\begin{array}{c}\text { Rectangular channel } \\
\text { Fixed lid }\end{array}$ & $\begin{array}{c}\text { Rectangular channel } \\
\text { Fixed lid }\end{array}$ & $\begin{array}{c}\text { Rectangular channel } \\
\text { Free surface }\end{array}$ \\
\hline Steady state & No & No & Yes \\
\hline Run duration $(\mathrm{min})$ & 10 & 5 & $60-120$ \\
\hline Flow depth, $D(\mathrm{~cm})$ & $0.3-0.9$ & $0.4-0.9$ & $0.13-0.31$ \\
\hline Flow width, $W(\mathrm{~cm})$ & 2.6 & 3.5 & 3 \\
\hline Reynolds number & 0.3 & $0.2-1.2$ & $300-1400$ \\
\hline Grain size, $d_{s}(\mu \mathrm{m})$ & 700 & 1100,2040 & $0.13-0.21$ \\
\hline Shields number, $\theta$ & $0.35-0.8$ & $0.22-1$ & 0.12 \\
\hline Threshold Shields, $\theta_{t}$ & 0.3 & 0.2 & $0.2-14$ \\
\hline Transport number, Tr & $2.4-71$ & $0.4-58$ & 344 \\
\hline
\end{tabular}

TABLE 1. Estimated parameter values for laminar bedload-transport experiments.

not investigated. The bedload instability is sensitive to boundary conditions; it persists in a rectangular pipe, but disappears when the flow is driven by a travelling lid.

Despite the simplicity of its driving mechanism, we could not find any report of this instability appearing in a laboratory experiment. This, of course, raises the question of its actual existence, but we suspect that the basic reason for its absence from the literature is that the aspect ratio of laboratory channels is usually too small to accommodate its growth. We speculate that this might not be fortuitous, as experimenters often wish the sediment bed to be invariant in the cross-stream direction.

At this point, we can only imagine an ideal set-up, specially designed to observe the bedload instability. Ideally, this set-up would involve a transparent rectangular pipe (as opposed to an open channel) to eliminate the fluctuations of the free surface. The pipe should be wider than the wavelength of the perturbation; an aspect ratio of approximately 20, for instance, should allow two parallel streaks to grow. The sediment and fluid discharges would be kept constant until the streaks, visualised with a laser beam, emerge. With the grains used by Seizilles et al. (2014), we expect an inverse growth rate of approximately 10 hours. If the bedload instability indeed appears in such an experiment, the critical transport number at which it does so would yield an estimate of the parameter $\gamma$, which represents the influence of gravity on the cross-stream transport of sediment.

Cross-stream fluxes of sediment shape the channel of alluvial rivers and, most probably, select their size (Ikeda et al. 1998). A reliable theory of bedload diffusion, tested against laboratory experiments, would therefore help us understand their morphology. Once such a framework is set, it will become a matter of numerical routines to explore it beyond linearity. A natural question to ask, then, will be whether the bedload instability grows 
as far as to split a broad channel into smaller ones, thus creating the precursor of a braided river (Stebbings 1963; Métivier et al. 2017).

We thank F. Métivier, J.A. Neufeld, D.H. Rothman, P. Szymczak, P. Gondret and S. Courrech du Pont for fruitful discussions. O.D. was partially funded by the Émergence(s) programme of the Mairie de Paris.

\section{REFERENCES}

ALlen, J.R.L. 1982 Sedimentary structures, their character and physical basis, , vol. 1. Elsevier. Andreotti, Bruno, Claudin, Philippe, Devauchelle, Olivier, Durán, Orencio \& Fourrière, ANToine 2012 Bedforms in a turbulent stream: ripples, chevrons and antidunes. Journal of Fluid Mechanics 690, 94-128.

Aussillous, Pascale, Chauchat, Julien, Pailha, Mickael, Médale, Marc \& Guazzelli, ELISABETH 2013 Investigation of the mobile granular layer in bedload transport by laminar shearing flows. Journal of Fluid Mechanics 736, 594-615.

Aussillous, Pascale, Zou, Zhenhai, Guazzelli, Élisabeth, Yan, Le \& Wyart, Matthieu 2016 Scale-free channeling patterns near the onset of erosion of sheared granular beds. Proceedings of the National Academy of Sciences p. 201609023.

BAgnold, R. 1973 The nature of saltation and of bedload transport in water. Proc. R. Soc. Lond. A 332, 473-504.

Bernal, JD \& MASON, J_ 1960 Packing of spheres: co-ordination of randomly packed spheres. Nature 188 (4754), 910.

ChARRU, François 2006 Selection of the ripple length on a granular bed sheared by a liquid flow. Physics of fluids $\mathbf{1 8}$ (12), 121508.

Charru, François, Andreotti, Bruno \& Claudin, Philippe 2013 Sand ripples and dunes. Annual Review of Fluid Mechanics 45, 469-493.

Charru, F., Mouilleron, H. \& Eiff, O. $2004 a$ Erosion and deposition of particles on a bed sheared by a viscous flow. Journal of Fluid Mech. 519, 55-80.

Charru, François, Mouilleron, Hélene \& Eiff, Olivier $2004 b$ Erosion and deposition of particles on a bed sheared by a viscous flow. Journal of Fluid Mechanics 519, 55-80.

Chen, Xiaoli, Ma, Jiming \& Dey, Subhasish 2009 Sediment transport on arbitrary slopes: Simplified model. Journal of Hydraulic Engineering 136 (5), 311-317.

Coleman, Stephen E \& Melville, Bruce W 1994 Bed-form development. Journal of hydraulic engineering $\mathbf{1 2 0}$ (5), 544-560.

Colombini, Marco 1993 Turbulence-driven secondary flows and formation of sand ridges. Journal of Fluid Mechanics 254, 701-719.

Colombini, Marco \& PARker, Gary 1995 Longitudinal streaks. Journal of Fluid Mechanics 304, 161-183.

Colombini, Marco, Seminara, Giovanni \& Tubino, Marco 1987 Finite-amplitude alternate bars. Journal of Fluid Mechanics 181, 213-232.

Devauchelle, O., Malverti, L., Lajeunesse, E., Josserand, C., Lagrée, Py \& MÉTIVIeR, F. 2010 Rhomboid beach pattern: A laboratory investigation. J. Geophys. Res 115, F02017.

Einstein, H. A. 1937 Bed load transport as a probability problem. In Sedimentation: 746 Symposium to Honor Professor H.A. Einstein, 1972. translation from 747 German of H.A. Einstein doctoral thesis. Originally presented to Federal Institute of Technology, Zurich, Switzerland, 1937, pp. C1 - C105.

EXNER, FELIX M 1925 Uber die wechselwirkung zwischen wasser und geschiebe in flussen. Akad. der Wiss in Wien, Math-Naturwissenschafliche Klasse, Sitzungsberichte, Abt IIa 134, 165-203.

Furbish, D.J., Haff, P.K., Roseberry, J.C. \& Schmeeckle, M.W. 2012 A probabilistic description of the bed load sediment flux: 1. theory. Journal of Geophysical Research 117 (F3), F03031.

IkedA, S., PARKer, G. \& Kimura, Y. 1998 Stable width and depth of straight gravel rivers with heterogeneous bed materials. Water Resour. Res. 24, 713-722. 
KARCZ, IAAKOV 1967 Harrow marks, current-aligned sedimentary structures. The Journal of Geology 75 (1), 113-121.

KEnNEDY, John F 1963 The mechanics of dunes and antidunes in erodible-bed channels. Journal of Fluid mechanics 16 (4), 521-544.

Kovacs, Agnes \& Parker, Gary 1994 A new vectorial bedload formulation and its application to the time evolution of straight river channels. Journal of fluid Mechanics 267, 153-183.

Lajeunesse, E., Malverti, L. \& Charru, F. 2010 Bedload transport in turbulent flow at the grain scale: experiments and modeling. J. Geophys. Res. Earth Surface 115, F04001, doi:10.1029/2009JF001628.

Lobkovsky, A.E., Orpe, A.V., Molloy, R., Kudrolli, A. \& Rothman, D.H. 2008 Erosion of a granular bed driven by laminar fluid flow. Journal of Fluid Mechanics 605 (1), 47-58.

Mclelland, Stuart J, Ashworth, Philip J, Best, James L \& Livesey, John R 1999 Turbulence and secondary flow over sediment stripes in weakly bimodal bed material. Journal of hydraulic engineering 125 (5), 463-473.

Métivier, F., Lajeunesse, E. \& Devauchelle, O. 2017 Laboratory rivers: Lacey's law, threshold theory, and channel stability. Earth Surface Dynamics 5 (1), 187-198.

Meyer-Peter, E. \& Müller, R. 1948 Formulas for bed-load transport. In Proceedings, 2nd Congress, International Association of Hydraulic Research (ed. Sweden Stockholm), pp. 39-64.

Nikora, Vladimir, Habersack, Helmut, Huber, Thomas \& McEwan, Ian 2002 On bed particle diffusion in gravel bed flows under weak bed load transport. Water Resources Research 38 (6), 17-1.

Ouriemi, Malika, Aussillous, Pascale \& Guazzelli, Elisabeth 2009 Sediment dynamics. part 1. bed-load transport by laminar shearing flows. Journal of Fluid Mechanics 636, 295-319.

Ouriemi, Malika, Aussillous, Pascale, Medale, Marc, Peysson, Yannick \& Guazzelli, Élisabeth 2007 Determination of the critical shields number for particle erosion in laminar flow. Physics of Fluids 19 (6), 061706.

PARKER, GARY 1976 On the cause and characteristic scales of meandering and braiding in rivers. Journal of fluid mechanics $\mathbf{7 6}$ (3), 457-480.

Seizilles, G., Lajeunesse, E., Devauchelle, O. \& Bak, M. 2014 Cross-stream diffusion in bedload transport. Phys. of Fluids 26 (1), 013302.

Seminara, G 2010 Fluvial sedimentary patterns. Annual Review of Fluid Mechanics 42, 43-66.

SHIELDS, A. S. 1936 Anwendung der aehnlichkeitsmechanik und der turbulenzforschung auf die geschiebebewegung. Mitteilung der Preussischen Versuchsanstalt fur Wasserbau und Schiffbau 26.

Stebbings, J. 1963 The shapes of self-formed model alluvial channels. Proceedings of the Institution of Civil Engineers 25 (4), 485-510, arXiv: https://doi.org/10.1680/iicep.1963.10544.

Vanderwel, Christina \& Ganapathisubramani, Bharathram 2015 Effects of spanwise spacing on large-scale secondary flows in rough-wall turbulent boundary layers. Journal of Fluid Mechanics $\mathbf{7 7 4}$.

Willingham, David, Anderson, William, Christensen, Kenneth T \& Barros, Julio M 2014 Turbulent boundary layer flow over transverse aerodynamic roughness transitions: induced mixing and flow characterization. Physics of Fluids 26 (2), 025111.

Yamasaka, Masashige, Ikeda, Syunsuke \& Kizaki, Shigeru 1987 Lateral sediment transport of heterogeneous bed materials. Doboku Gakkai Ronbunshu 1987 (387), 105114. 